\title{
Longitudinal Changes in Brain Metabolic Activity after Withdrawal from Escalation of Cocaine Self-Administration
}

\author{
Céline Nicolass $^{1,2}$, Clovis Tauber ${ }^{3}$, François-Xavier Lepelletier ${ }^{3}$, Sylvie Chalon ${ }^{3}$, Pauline Belujon ${ }^{1,2}$, \\ Laurent Galineau ${ }^{3,4}$ and Marcello Solinas ${ }^{*, 1,2,4}$ \\ IINSERM, UI 084, Poitiers, France; ' Université de Poitiers, UI 084, Poitiers, France; ${ }^{3}$ UMR INSERM U930, Université François Rabelais de Tours, \\ Tours, France
}

\begin{abstract}
The chronic and relapsing nature of addiction suggests that drugs produce persistent adaptations in the brain that make individuals with drug addiction particularly sensitive to drug-related cues and stress and incapable of controlling drug-seeking and drug-taking behavior. In animal models, several long-lasting neuroadaptations have been described. However, few studies have used brain-imaging techniques to provide a complete picture of brain functioning in the course of withdrawal from cocaine. In this study, we allowed rats to self-administer cocaine under short-access (I-h/day) or long-access (6-h/day) conditions and used 2-deoxy-2- $\left({ }^{18} \mathrm{~F}\right)$ fluoro-d-glucose $\left({ }^{18} \mathrm{FDG}\right)$ positron emission tomography scanning to investigate the longitudinal changes in metabolic activity I and 4 weeks after discontinuation of cocaine self-administration. We found that compared to naive rats, both long-access and short-access rats showed significant disruptions in basal brain metabolic activity. However, compared to short-access, long-access rats showed more intense, and long-lasting neuroadaptations in a network of brain areas. In particular, abstinence from extended access to cocaine was associated with decreased metabolic activity in the anterior cingulate cortex, the insular cortex, and the dorsolateral striatum, and increased metabolic activity in the mesencephalon, amygdala, and hippocampus. This pattern is strikingly similar to that described in humans that has led to the proposal of the Impaired Response Inhibition and Salience Attribution model of addiction. These results demonstrate that extended access to cocaine leads to persistent neuroadaptations in brain regions involved in motivation, salience attribution, memory, stress, and inhibitory control that may underlie increased risks of relapse.
\end{abstract}

Neuropsychopharmacology (2017) 42, 1981-1990; doi:I0.1038/npp.2017.109; published online 21 June 2017

\section{INTRODUCTION}

Brain imaging approaches are powerful tools to identify differences in brain structure and function in individuals with drug addiction compared to healthy controls (Goldstein and Volkow, 2002; Volkow et al, 1997b, 2003). Some of these modifications persist long after discontinuation of drug use and may underlie the persistent risk of relapse over time whereas others appear recovered after withdrawal from the drug (Goldstein and Volkow, 2002; Parvaz et al, 2011). For example, using 2-deoxy-2- $\left[{ }^{18} \mathrm{~F}\right]$ fluoro-d-glucose $\left({ }^{18} \mathrm{FDG}\right)$ positron emission tomography (PET) imaging, it has been found that during early withdrawal, individuals with cocaine addiction show global increases in functional brain activity whereas after protracted periods of withdrawal, brain activity decreases compared to naive subjects (Goldstein and

\footnotetext{
*Correspondence: Dr M Solinas, Neurobiology and Neuropharmacology of Addiction Team, Laboratory of Experimental and Clinical Neurosciences, INSERM UI 084, University of Poitiers, Bât. B36-Pôle Biologie Santé, I, rue Georges Bonnet-BP 633, Poitiers 86022, France, Tel: +33549 366343, Fax: +335494540।4,

E-mail: marcello.solinas@univ-poitiers.fr

${ }^{4}$ These authors contributed equally to this work.

Received 23 January 2017; revised 22 May 2017; accepted 24 May 2017; accepted article preview online 29 May 2017
}

Volkow, 2002; Parvaz et al, 2011; Volkow et al, 1991, 1997b). Neuroimaging studies have been instrumental to the understanding of the brain changes associated with addiction (Goldstein and Volkow, 2002; Koob and Volkow, 2010).

Whereas brain imaging in humans have greatly contributed to our understanding of the neurobiological dysfunctions associated with drug addiction, rodent models could be invaluable tools to rapidly test novel hypotheses that could be then translated back to humans. In animals, several studies have used brain imaging to investigate the changes in brain activity induced by drugs (Caprioli et al, 2013; Gould et al, 2014; Hammer et al, 1993; Hanlon et al, 2013; Howell, 2008; Macey et al, 2004). Using functional magnetic resonance imaging (fMRI), Gozzi et al found that after 10 days of abstinence rats with a history of extended access to cocaine self-administration show decreases in basal cerebral blood flow in fronto-cortical regions such as the orbitofrontal (OFC), prefrontal and anterior cingulate (ACC) cortices, and the nucleus accumbens (NAc) (Gozzi et al, 2011). In another study, Calipari et al used ex vivo $2-\left[{ }^{14} \mathrm{C}\right]$ deoxyglucose methods and found a decrease in the activity of several brain areas $48 \mathrm{~h}$ after 5 days of extended access to cocaine self-administration, including the NAc and the ACC (Calipari et al, 2013). However until now, no longitudinal study has been performed to assess changes in brain activity 
that occur after short and long periods of abstinence from cocaine. Importantly, investigating changes in brain activity after long periods of withdrawal could enable obtaining information about brain recovery processes and about mechanisms underlying incubation of drug craving (Grimm et al, 2001; Hanlon et al, 2013; Pickens et al, 2011). In the present study, we used PET with ${ }^{18}$ FDG to evaluate metabolic activity changes after short (1 week) and long (4 weeks) periods of abstinence from cocaine in rats with a history of cocaine self-administration using the escalation model (Ahmed and Koob, 1998). In this model, rats are given either short-access $(\mathrm{ShA})$ or long-access $(\operatorname{LgA})$ to cocaine self-administration for several weeks, allowing comparing recreational use in ShA rats to extended addiction-like escalated intake in $\operatorname{LgA}$ rats. Thus, the comparison of the brain metabolic maps obtained for ShA, $\operatorname{LgA}$ and naive rats, may provide insights into the short- and long-term cerebral metabolic changes after withdrawal after exposure to different regimens of cocaine intake.

\section{MATERIALS AND METHODS}

For detailed material and methods see Supplementary Information.

\section{Subjects and Housing Conditions}

Adult male Sprague-Dawley rats (Janvier Labs, France), experimentally naive at the beginning of the study, were used in this study. All experiments were conducted in accordance with European Union directives (2010/63/EU) for the care of laboratory animals and approved by the local ethics committees (COMETHEA).

\section{General Experimental Design}

Our general experimental design is schematized in Figure 1. During the first 7 days, rats were allowed to self-administer cocaine in 2-h training sessions. On the 8th day, rats were divided into two groups: one group had access to cocaine for $1 \mathrm{~h}(\mathrm{ShA}, n=8)$ and the other group had access to cocaine for $6 \mathrm{~h}(\operatorname{LgA}, n=8)$ to 20 sessions. At the end of the last selfadministration session (day 20), rats were transferred from the animal facility at the University of Poitiers to the animal facility at University of Tours by an authorized transporter and underwent abstinence for 4 weeks. Metabolic imaging using ${ }^{18}$ FDG was performed in the same rats after 6-8 days (1 week) and 27-29 days (4 weeks) of abstinence. Naive rats of the same age and with analogous housing conditions were used as controls $(n=8)$.

\section{Brain Imaging}

Local uptake of ${ }^{18} \mathrm{FDG}$ reflects cerebral metabolic rates of glucose utilization and allows the investigation of regional brain metabolic status (Phelps et al, 1979; Sokoloff, 1977). Metabolic imaging using ${ }^{18} \mathrm{FDG}$ was performed under basal conditions. Rats were habituated to the PET experimental procedures for 4 days before each scan, and fasted overnight before each scan. The day of brain-imaging acquisition, awake rats were injected with ${ }^{18} \mathrm{FDG}(18.5 \mathrm{MBq} / 100 \mathrm{~g}$ i.p.; Cyclopharma, Tours), and placed in the habituation cage for $45 \mathrm{~min}$. Then, they were anesthetized using isoflurane $4 \%$ (Baxter, Maurepas, France), placed on a heating pad (Minerve, Esternay, France) and centered in the field of view of the Explore VISTA-CT microPET camera (GE Healthcare, Velizy, France). A CT-scan was performed for attenuation correction of PET images and a list-mode PET acquisition of $30 \mathrm{~min}$ started $60 \mathrm{~min}$ after ${ }^{18} \mathrm{FDG}$ injection. After data reconstruction using a 2-D OSEM algorithm, all images were co-registered and normalized for tissue activity in the whole brain. Quantitative results were expressed as mean \pm SD and were presented on Z-score maps. Analyses focused on brain areas known to be key nodes in addiction: the ACC, OFC, prelimbic (PrL), infralimbic (IL), insular and motor cortices, in addition to the dorsal striatum (DStr), NAc, ventral pallidum (VP), substantia nigra/ventral tegmental area (SN/VTA), amygdala (Amyg), and hippocampus (Hipp) (Volkow and Baler, 2014). It should be noted that areas that were too small to be identified using microPET ${ }^{18}$ FDG imaging were not included in our analysis.

\section{Statistical Analyses}

For self-administration, two-way repeated measures ANOVA with time as a within-subject factor and cocaine exposure ( $\operatorname{LgA}$ or $\mathrm{ShA}$ ) as a between-subject factor was

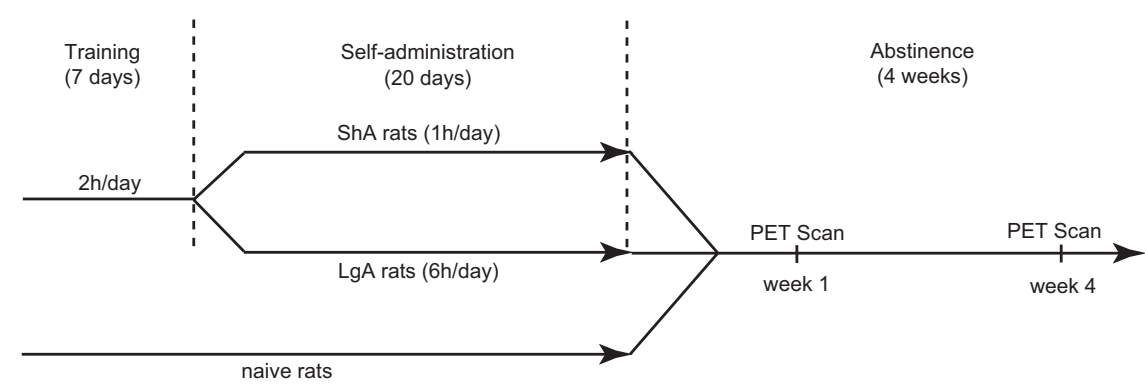

Figure I Experimental design. After 7 days of cocaine self-administration training (2-h/day), rats were assigned to two groups short access (ShA), which have access to cocaine for I-h/day and long access (LgA), which have access to cocaine for 6-h/day. At the end of the 20 days of cocaine self-administration, rats underwent a 4-week abstinence during which brain metabolic imaging using ${ }^{18}$ FDG was performed at I week and 4 weeks of abstinence. Naive rats were used as controls. 

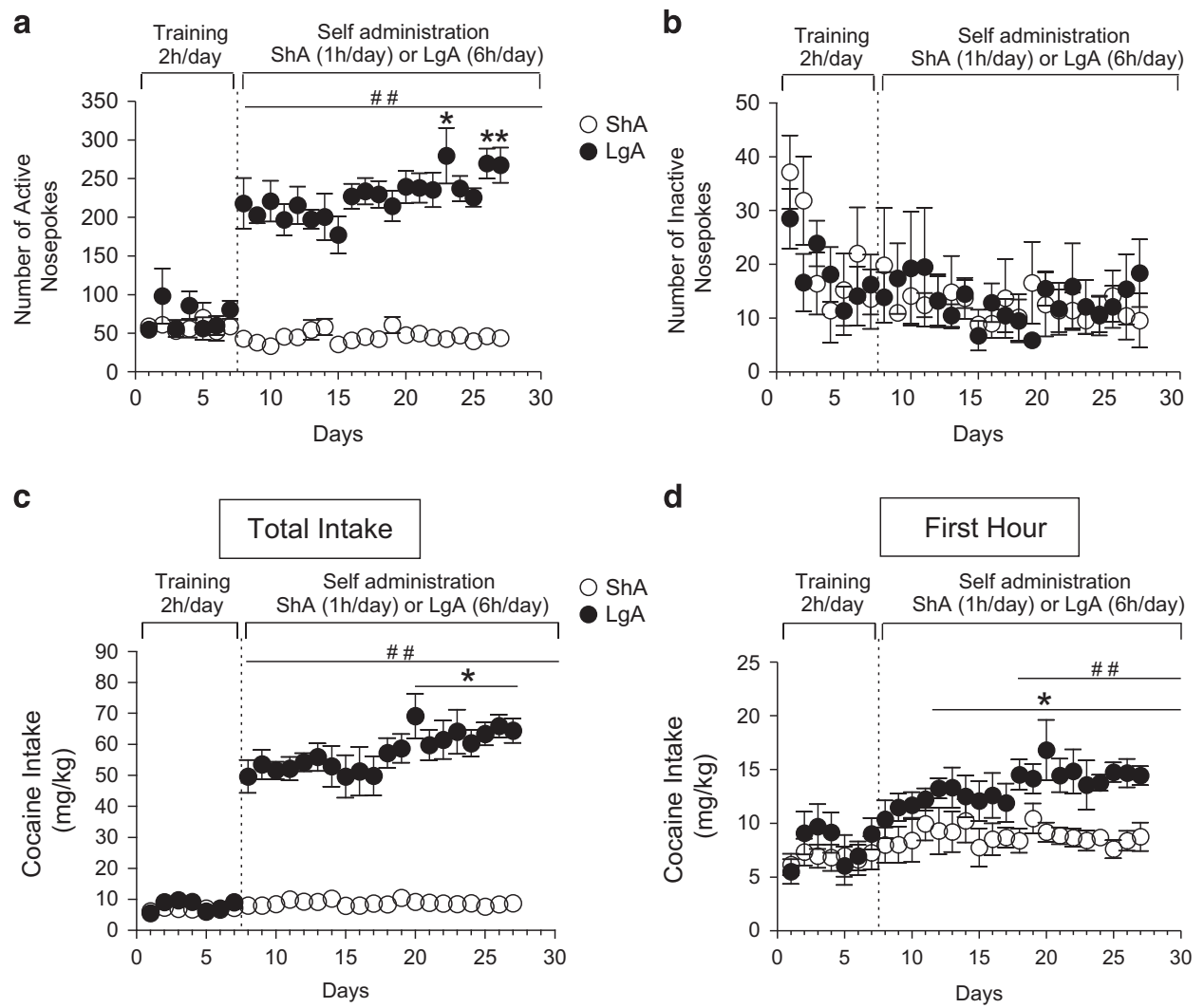

Figure 2 Cocaine self-administration training. Number of active (a) and inactive (b) nose-pokes, total cocaine intake (c) and cocaine intake during the first hour of sessions (d) during the training phase in which all animals had access to cocaine for $2 \mathrm{~h}$ (left part of the graphs) and during the escalation phase in which rats were divided into short access (ShA, $n=8, I$-h sessions) and long access ( $\mathrm{LgA}, n=8,6$-h sessions) groups (right part of the graphs). $* 0<0.05$ and *** $p<0.0$ I compared to session I of LgA self-administration, ${ }^{\# \#} p<0.000$ I LgA compared to ShA group.

used. Results showing significant overall changes were subjected to a Student-Newman-Keuls post hoc test.

For micro-PET data, a voxel-based analysis was used to assess the differences in cerebral ${ }^{18} \mathrm{FDG}$ uptake between the averaged brains of $\operatorname{LgA} / \mathrm{ShA} v s$ control rats at each stage of withdrawal. The regions of interest were derived from Schiffer's templates (Schiffer et al, 2006) using PMOD v3.2 software (PMOD Technologies Ltd, Switzerland) and applied to Z-score maps to obtain the Z-score values in these areas. Inter-group comparison was performed using a two-tail unpaired Student $t$-test. Intra-group comparisons were performed using a twotail paired $t$-test to reveal within-rat differences over the course of cocaine withdrawal. Differences were considered significant when $p<0.05$.

\section{RESULTS}

\section{Self-Administration}

Cocaine self-administration started with seven 2-h sessions (Figure 2, left in all graphs a-d). After the initial training, rats were divided into two groups and were allowed to selfadminister cocaine for 1-h (ShA) and 6-h ( LgA) for 20 additional sessions (Figure 2, right in all graphs a-d). In the ShA group, active nose-pokes (Figure 2a) and cocaine intake (Figure 2c) during the 20 sessions were stable whereas in the LgA group, the number of active nose-pokes (Figure 2a) and cocaine intake (Figure 2c) significantly increased over time.
Figure $2 \mathrm{~d}$ shows the cocaine intake during the first hour of access to the drug in ShA and LgA. During the first 7 days of self-administration ShA and LgA rats had similar levels of cocaine intake. In the ShA group the cocaine intake was stable over time, from $8.0 \pm 1.6$ at day 8 to $8.8 \pm 1.3 \mathrm{mg} / \mathrm{kg}$ of cocaine at day 27 . In contrast, in the LgA group, the cocaine intake significantly escalated over time, from $10.4 \pm 1.7$ at day 8 to $14.8 \pm 0.9 \mathrm{mg} / \mathrm{kg}$ of cocaine at day 27 . Statistical analysis revealed a significant effect of time $[\mathrm{F}(20280)=2.83$, $p<0.0001]$ and a significant GroupXTime interaction $[\mathrm{F}(20280)=2.14, p<0.01]$.

\section{Brain Metabolic Activity in Long-Access and Short-Access Rats During Abstinence in Areas Involved in Reward, Salience, Motivation and Drive}

Nucleus accumbens. In the NAc, ${ }^{18}$ FDG uptake was not altered in the ShA group (Figure 3) but was reduced in $\operatorname{LgA}$ rats $v s$ controls after short periods of abstinence (Table 1) and recovered after 4 weeks of abstinence (Figure 4, Tables 1 and 2). The direct comparison between LgA and ShA rats confirmed that the metabolic activity of the NAc was reduced after 1 but not after 4 weeks of abstinence in $\operatorname{LgA}$ rats compared to ShA rats (Figure 5, Table 1).

Ventral pallidum. No significant differences between groups were found in the VP at any time point (Figures 3 and 4). 

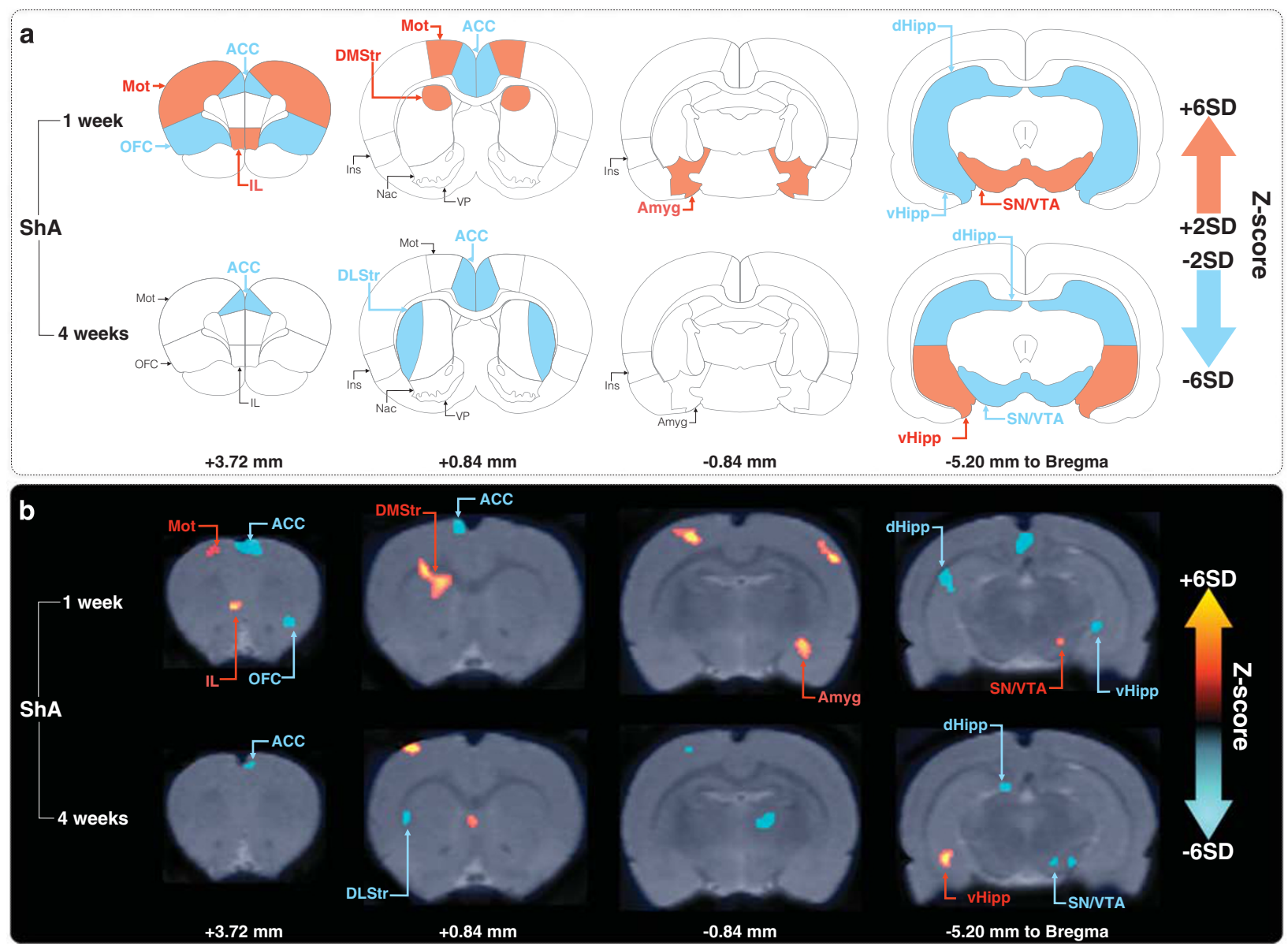

Figure 3 Changes in metabolic activity in ShA rats compared to naive controls. (a) Summary of the significant increases (red) and decreases (blue) in ${ }^{18} \mathrm{FDG}$ uptake observed in ShA rats vs controls ( $n=8 /$ group) after I week (upper part) and 4 weeks (lower part) of cocaine withdrawal presented on representative coronal plates of the Paxinos and Watson atlas (Student's two-tailed $t$-test; $p<0.0 \mathrm{I}$ ). (b) Examples of the significant differences in ${ }^{18} \mathrm{FDG}$ uptake observed between ShA and control rats after I week (upper part) and 4 weeks (lower part) of cocaine abstinence presented on coronal images of Z-score maps fused with an MRI template (increases in ${ }^{18}$ FDG uptake from dark red to yellow, decreases in ${ }^{18}$ FDG uptake from black to light blue; Student's two-tailed $t$-test; $p<0.0 \mathrm{I}$ ). ACC, anterior cingulate cortex; Amyg, amygdala; DHipp, dorsal hippocampus; DMStr, dorsomedial striatum; DLStr, dorsolateral striatum; IL, infralimbic cortex; Ins, insula; Mot, motor cortex; NAc, nucleus accumbens; OFC, orbitofrontal cortex; PrL, prelimbic cortex; SN/VTA, substantia nigra/ventral tegmental area; VHipp, ventral hippocampus; VP, ventral pallidum.

Dorsal striatum. In the DStr, ${ }^{18} \mathrm{FDG}$ uptake was increased in both ShA and LgA rats in the medial part (DMStr) after short periods of abstinence (Table 1) and decreased in the lateral part (DLStr) after long periods of abstinence compared to controls (Figures 3 and 4, Table 1). Thus, the metabolic activity was restored in the DMStr and decreased in the DLStr during cocaine abstinence in both groups (Table 2). Importantly, direct comparison of $\operatorname{LgA}$ to ShA rats showed that the metabolic activity of the DLStr was similar after 1 week of abstinence but it was lower in LgA compared to ShA rats after 4 weeks (Figure 5, Table 1), suggesting that extended access to cocaine produces quantitatively bigger disruptions in the activity of this brain area.

Substantia nigra/ventral tegmental area. In the SN/VTA, increased ${ }^{18}$ FDG uptake was observed after 1 week of abstinence in both ShA and LgA rats compared to controls (Figures 3, and 4, Table 1), but this effect was stronger in LgA than in ShA rats (Figure 5, Table 1). After 4 weeks of abstinence, increased ${ }^{18} \mathrm{FDG}$ uptake was still observed and it was significantly stronger in the SN/VTA of LgA rats (Figure 3, Tables 1 and 2), whereas a decreased uptake was observed in ShA rats (Figure 4, Tables 1 and 2). Direct comparison of $\operatorname{LgA}$ and ShA rats confirmed that the metabolic activity of the SN/VTA was higher in LgA compared to ShA rats (Figure 5, Table 1).

Orbitofrontal cortex. In the OFC, a decreased metabolic activity compared to controls was found in ShA rats after 1 week of abstinence but this returned to control levels after 4 weeks of abstinence (Figure 3, Tables 1 and 2). No change in the metabolic activity of this region was detected in LgA rats (Figure 4). Direct LgA/ShA comparison showed that the metabolic activity in the OFC of $\operatorname{LgA}$ rats was increased compared to ShA rats after 1 week of abstinence (Figure 5, Table 1) but this effect was due to metabolic hypoactivity in ShA rats rather than a change compared to control. 
Table I Statistical Significances for Inter-Group Comparisons

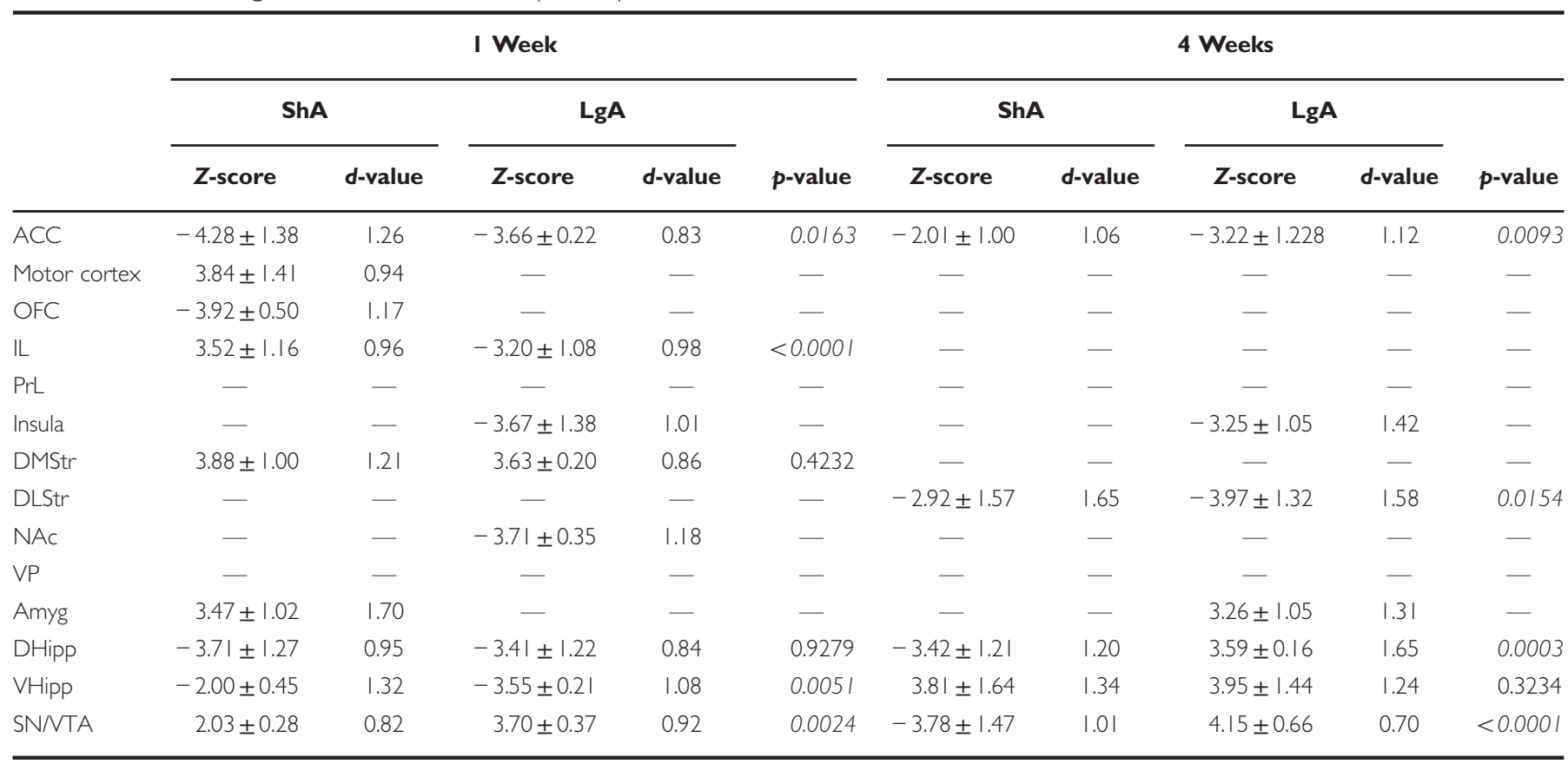

Abbreviations: Amyg, amygdala; ACC, cingulate cortex; DHipp, dorsal hippocampus; DMStr, dorsomedial striatum; DLStr, dorsolateral striatum; IL, infralimbic cortex; LgA, long-access; NAc, nucleus accumbens; OFC, orbitofrontal cortex; PrL, prelimbic cortex; ShA, short-access; SN/NTA, substantia nigra/ventral tegmental area; VHipp, ventral hippocampus; VP, ventral pallidum. The Z-score and $d$-values are presented for each significant differences observed between ShA and LgA rats vs controls after I or 4 weeks of cocaine abstinence. $P$-values of unpaired $t$-test also show the differences between $L g A$ and ShA at each withdrawal stage. It should be noticed that $p$-values were only calculated when both ShA and LgA showed a significant effect (Z-score $>2$ ). Italic font indicates significant p-values.

Motor cortex. In the Motor cortex, increased ${ }^{18}$ FDG uptake was found in ShA (Figure 3, Table 1) but not $\operatorname{LgA}$ rats compared to controls after 1 week of abstinence (Figure 4). However, the metabolic activity of this region returned to control levels after 4 weeks of abstinence (Figures 3 and 4, Table 2). Direct LgA/ShA comparison highlighted a lower metabolic activity in the Motor cortex of $\operatorname{LgA}$ compared to ShA rats after 1 week of abstinence (Figure 5, Table 1) but this effect was due to metabolic hyperactivity in ShA rats rather than a change compared to controls.

\section{Brain Metabolic Activity in Long-Access and Short-Access Rats after Abstinence in Areas Involved in Inhibitory Control, Executive Functions, and Interoception}

Prelimbic and infralimbic cortices. No change in the metabolic activity of the PrL was observed in any group at any time point (Figures 3 and 4 ). On the other hand, the IL shows increases in ${ }^{18}$ FDG uptakes in ShA (Figure 3, Table 1) and decreases in LgA rats (Figure 3, Table 1) after 1 week of abstinence but both modifications disappeared after 4 weeks of abstinence (Figures 3 and 4, Table 2). Direct LgA/ShA comparison confirmed that the IL of $\operatorname{LgA}$ rats show a hypoactive metabolism compared to ShA rats specifically after a short period of abstinence (Figure 5, Table 1).

Anterior cingulate cortex. The ${ }^{18}$ FDG uptake was reduced in the ACC in both ShA and LgA rats compared to controls after both short and long periods of abstinence (Figures 3 and 4, Table 1). Whereas the decreases observed in LgA rats persisted overtime, in ShA rats the effect was significantly weaker after 28 days of cocaine abstinence (Table 2) indicating some level of recovery in this group. When we compared directly $\operatorname{LgA}$ to ShA rats, we found that the hypoactive metabolism was more pronounced in ShA rats after 1 week of abstinence (Table 1), while the contrary was observed after 4 weeks of abstinence with LgA rats showing less activity of the ACC compared to ShA rats (Figure 5, Table 1). This suggests that extended self-administration of cocaine produced more intense and more persistent disruptions in the activity of this brain area.

Insular cortex. In the insula, the ${ }^{18}$ FDG uptake was reduced in $\operatorname{LgA}$, but not in ShA rats after short and long periods of abstinence (Figures 3 and 4, Table 1). Direct LgA/ ShA comparison confirmed that the metabolic activity in this area was consistently lower in LgA compared to ShA rats (Figure 5, Table 1). The effect observed in LgA rats persisted over the course of cocaine withdrawal (Table 2).

\section{Brain Metabolic Activity in Long-Access and Short-Access Rats after Abstinence in Areas Involved in Contextual and Emotional Memories}

Hippocampus. After short periods of abstinence, metabolic activity was reduced in both ShA and LgA rats in the dorsal part of the Hipp (DHipp) and in the ventral part (VHipp; Figures 3 and 4, Table 1).

After 4 weeks of abstinence, in ShA rats the metabolic activity of the DHipp was still reduced (Table 1) and not significantly different from what was observed after 1 week 

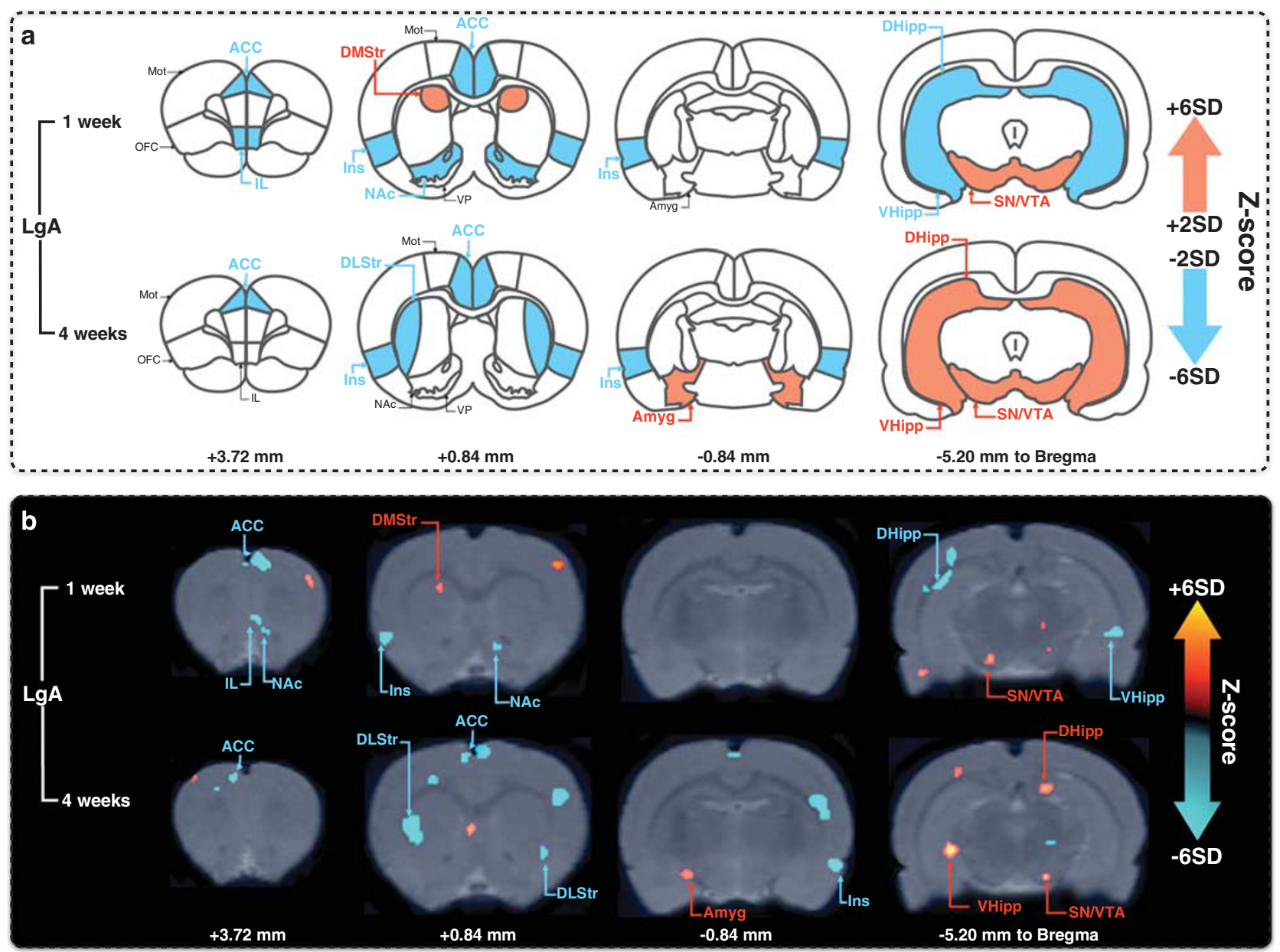

Figure 4 Changes in metabolic activity in LgA rats compared to naive controls. (a) Summary of the significant increases (red) and decreases (blue) in ${ }^{18}$ FDG uptake observed in LgA rats vs controls ( $n=8 /$ group) after I week (upper part) and 4 weeks (lower part) of cocaine abstinence presented on representative coronal plates of the Paxinos and Watson atlas (Student's two-tailed t-test; $p<0.01$ ). (b) Examples of the significant differences in ${ }^{18}$ FDG uptake observed between LgA and control rats after I week (upper part) and 4 weeks (lower part) of cocaine abstinence presented on coronal images of Z-score maps fused with an MRI template (increases in ${ }^{18}$ FDG uptake from dark red to yellow, decreases in ${ }^{18}$ FDG uptake from black to light blue; Student's two-tailed t-test; $p<0.0$ I). ACC, anterior cingulate cortex; Amyg, amygdala; DHipp, dorsal hippocampus; DMStr, dorsomedial striatum; DLStr, dorsolateral striatum; IL, infralimbic cortex; Ins, insula; Mot, motor cortex; NAc, nucleus accumbens; OFC, orbitofrontal cortex; PrL, prelimbic cortex; SN/VTA, substantia nigra/ventral tegmental area; VHipp, ventral hippocampus; VP, ventral pallidum.

(Table 2), whereas the VHipp became hyperactive (Tables 1 and 2). In contrast, both the DHipp and the VHipp became hyperactive in $\mathrm{LgA}$ rats compared to controls after 4 weeks of abstinence (Figure 4, Tables 1 and 2). When we compared directly $\operatorname{LgA}$ to ShA rats, the metabolic activity of the VHipp was lower in $\mathrm{LgA}$ rats compared to ShA rats after 1 week of abstinence suggesting that hypoactivity of this region was more pronounced in $\operatorname{LgA}$ than in ShA rats (Figure 5, Table 1). In addition, after 4 weeks of abstinence, the metabolic activity of the DHipp was higher in LgA compared to ShA rats (Figure 5, Table 1).

Amygdala. In ShA rats, the metabolic activity of the Amyg, was increased compared to controls after short periods of abstinence (Table 1) but recovered after 4 weeks of abstinence (Figure 3, Table 2). Conversely, in LgA rats the metabolic activity of the Amyg was normal after short periods of abstinence but increased after 4 weeks of abstinence (Figure 4, Tables 1 and 2). Direct comparison of
$\operatorname{LgA}$ and ShA rats, confirmed that the metabolic activity of the Amyg in LgA rats was lower compared to ShA rats after 1 week of abstinence but became higher after 4 weeks of abstinence (Figure 5, Table 1).

\section{DISCUSSION}

We show that voluntary intake of cocaine produces changes in basal brain metabolic activity that depends on the intensity of cocaine self-administration and on the duration of abstinence. Indeed, escalation of cocaine selfadministration produces cerebral changes that are quantitatively and qualitatively different from those found after ShA cocaine self-administration. Importantly, the neuroadaptations found in this study are consistent with data described in humans and support the idea that cocaine exposure is associated with specific disruptions in interconnected mesostriato-cortical, limbic and fronto-cortical circuits that are involved in reward, motivation, salience attribution, 
Table 2 Statistical Significances for Intra-group Comparisons

\begin{tabular}{|c|c|c|c|c|c|c|c|c|c|c|}
\hline & \multicolumn{5}{|c|}{ ShA } & \multicolumn{5}{|c|}{ LgA } \\
\hline & I Week & 4 Weeks & $p$-value & DF & $t$ & I Week & 4 Weeks & $p$-value & DF & $t$ \\
\hline Motor cortex & $3.84 \pm 1.41$ & - & $<0.0001$ & 7 & 12.200 & - & - & - & - & - \\
\hline OFC & $-3.92 \pm 0.50$ & - & 0.0454 & 7 & 2.518 & - & - & - & - & - \\
\hline Insula & - & - & - & - & - & $-3.67 \pm 1.38$ & $-3.25 \pm 1.05$ & 0.1188 & 7 & 1.777 \\
\hline DMStr & $3.88 \pm 1.00$ & - & 0.0105 & 7 & 3.460 & $3.63 \pm 0.20$ & - & 0.0372 & 7 & 2.567 \\
\hline DLStr & - & $-2.92 \pm 1.57$ & 0.0455 & 7 & 2.429 & - & $-3.97 \pm 1.32$ & 0.0374 & 7 & 2.562 \\
\hline $\mathrm{NAc}$ & - & - & - & - & - & $-3.7 \mid \pm 0.35$ & - & $<0.0001$ & 7 & 12.410 \\
\hline VP & - & - & - & - & - & - & - & - & - & - \\
\hline VHipp & $-2.00 \pm 0.45$ & $3.81 \pm 1.64$ & 0.0002 & 7 & 6.831 & $-3.55 \pm 0.21$ & $3.95 \pm 1.44$ & 0.0042 & 7 & 4.489 \\
\hline SN/NTA & $2.03 \pm 0.28$ & $-3.78 \pm 1.47$ & $<0.0001$ & 7 & 9.263 & $3.70 \pm 0.37$ & $4.15 \pm 0.66$ & 0.0310 & 7 & 2.803 \\
\hline
\end{tabular}

Abbreviations: Amyg, amygdala; ACC, cingulate cortex; DHipp, dorsal hippocampus; DMStr, dorsomedial striatum; DLStr, dorsolateral striatum; IL, infralimbic cortex; LgA, long-access; NAc, nucleus accumbens; OFC, orbitofrontal cortex; PrL, prelimbic cortex; ShA, short-access; SN/NTA, substantia nigra/ventral tegmental area; VHipp, ventral hippocampus; VP, ventral pallidum. The Z-score, $t$, DF and $p$-values of paired $t$-test are presented for each significant differences observed during cocaine abstinence within ShA and LgA rats. Italic font indicates significant $p$-values.

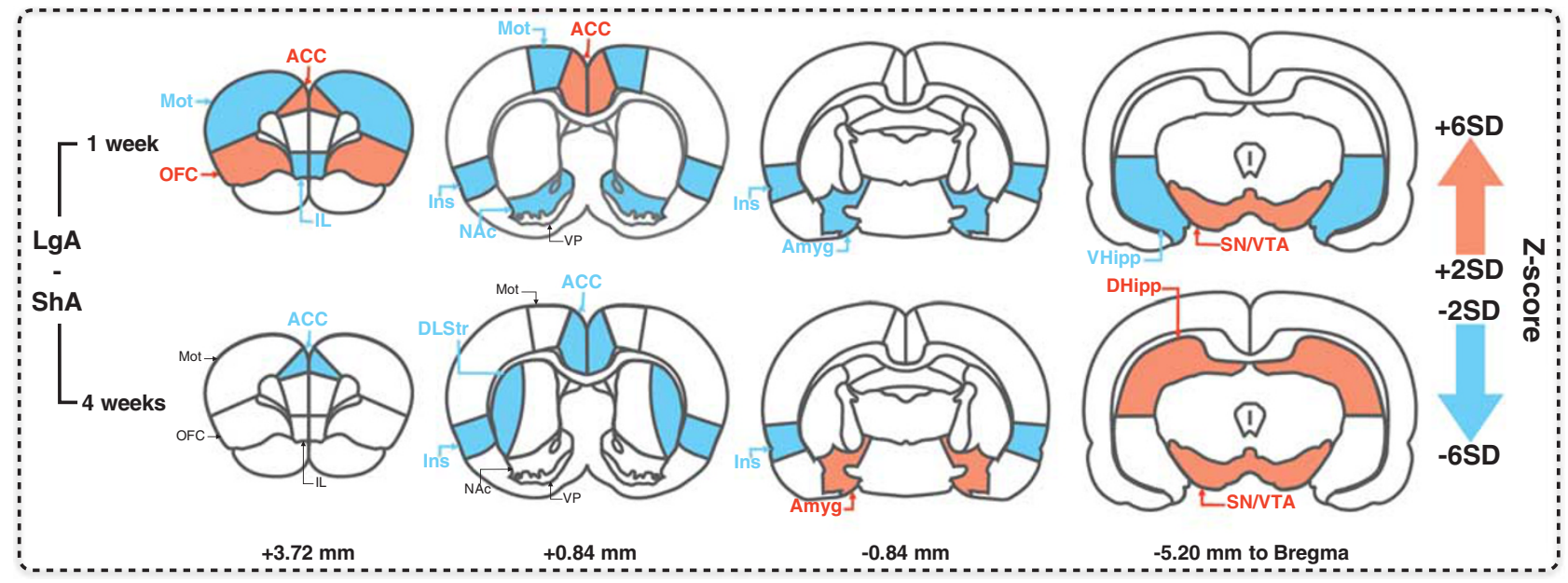

Figure 5 Comparisons of the changes in metabolic activity in LgA vs ShA rats. Summary of the significant increases (red) and decreases (blue) in ${ }^{18}$ FDG uptake observed in LgA vs ShA rats ( $n=8 /$ group) after I week (upper part) and 4 weeks (lower part) of cocaine abstinence presented on representative coronal plates of the Paxinos and Watson atlas (Student's two-tailed t-test; $p<0.0$ I).

executive control, stress reactivity and negative affect (Goldstein and Volkow, 2002; Koob and Volkow, 2010; Parvaz et al, 2011; Volkow and Baler, 2014; Volkow et al, 2003).

\section{Longitudinal Brain Dysregulations During Abstinence from Cocaine Self-Administration}

Several animal studies have investigated the changes in brain functioning associated with drug exposure (Caprioli et al, 2013; Gould et al, 2014; Hammer et al, 1993; Hanlon et al, 2013; Howell, 2008; Macey et al, 2004) but only two studies have focused on withdrawal from extended access to cocaine using brain imaging in rats (Calipari et al, 2013; Gozzi et al, 2011). Whereas those studies were limited to the early consequences of cocaine withdrawal on brain activity, here we investigated longitudinally both early and long-term changes in metabolic activity related to cocaine abstinence in rats exhibiting two different patterns of cocaine voluntary intake (stable low intake and escalated high intake). Although the direct comparison of the results is complicated because of the different experimental protocols and imaging approaches used (fMRI vs PET), a common finding in all studies is that cocaine-exposed rats displayed decreased 
metabolic activities in the ACC, medial prefrontal cortex and the NAc after short periods of withdrawal (Calipari et al, 2013; Gozzi et al, 2011). It is important to note that some of these adaptations were found both after limited and extended access to cocaine. However, the intensity of metabolic modification was significantly higher in LgA than in ShA. In addition, whereas some brain regions show spontaneous recovery of normal metabolic activity after protracted abstinence, in LgA rats, a few brain structures (the DStr, the ACC, the SN/VTA, the Amyg, the Hipp and the insula) show persistent changes in metabolic activity that may contribute to long-lasting risks of relapse. These results are consistent with separate previous studies that have shown that withdrawal from cocaine self-administration is associated with neuroadaptations in the DStr (Hearing et al, 2008; Pomierny-Chamiolo et al, 2015), ACC (PomiernyChamiolo et al, 2015; Zavala et al, 2007; Zorrilla et al, 2001), VTA (mostly with no change in the SN (Arroyo et al, 2000; Chen et al, 2008; Grimm et al, 2003; Lu et al, 2003), Amyg (Lee et al, 2013; Lu et al, 2005a; Zorrilla et al, 2001) and Hipp (Garcia-Fuster et al, 2012; Noonan et al, 2008; PomiernyChamiolo et al, 2015; Thompson et al, 2004). Importantly, each of these regions has been shown to be involved in cocaine craving and relapse in animal models (Cosme et al, 2015; Fuchs et al, 2005, 2006; Grimm and See, 2000; Lu et al, 2005b; McFarland and Kalivas, 2001; Torregrossa et al, 2013). Thus, these results suggest that extended access cocaine self-administration is associated with simultaneous dysregulations in interconnected networks that can lead to increased risks of relapse.

\section{Similarities between Rat and Human Studies}

Over a decade ago, on the basis of clinical and brain-imaging data in humans, Goldstein and Volkow proposed the Impaired Response Inhibition and Salience Attribution (I-RISA) model of addiction that postulates that addiction is the result of a change in the balance of different functions and in the activity of corresponding brain areas (Goldstein and Volkow, 2002). In this model, the six main functions involved in drug addiction are: (1) reward/saliency involving the NAc, the VP and the dopaminergic system; (2) memory/ learning/habits involving the DStr, the Amyg and the Hipp; (3) inhibitory control/executive functions involving the prefrontal cortex and the ACC; (4) motivation/drive involving the OFC, the motor cortex and the dopaminergic system (SN/VTA); (5) interoception involving the insula and ACC and (6) aversion involving the Amyg (Goldstein and Volkow, 2002; Koob and Volkow, 2010; Parvaz et al, 2011; Volkow and Baler, 2014; Volkow et al, 2003). In particular, in this model, reductions in the activity of prefrontal and frontal cortical areas induce dysregulated top-down processes ultimately leading to difficulties in controlling and inhibiting drug-seeking behavior and increased risks of relapse (for review see Goldstein and Volkow (2002)). For example, long-term abstinent cocaine abusers (more than 6 weeks) show frontal hypo-metabolism (Volkow et al, 1992) and this disruption is supposed to favor compulsive drug taking (Volkow et al, 1993). This hypo-metabolism is associated with decreased dopamine D2 receptor availability (Volkow et al, 1990, 1993), reflecting a dysregulation of dopamine receptors following alterations in the activity of the dopaminergic system, described in detoxified cocaine abusers (Volkow et al, 1997a, 1997c). Our data demonstrate that rats that have extended access to cocaine show a profile of brain metabolic activity that is consistent with this model. In fact, we found a persistent reduction in the metabolic activity of ACC associated with an increase in the activity of the SN/VTA and a decrease in the activity of the DStr, which could be secondary to hyperactivity of the dopaminergic system and activation of dopamine receptors. In addition, after long periods of abstinence, we found that the Amyg and Hipp are hyperactive which is consistent with the critical role of memory, negative affect and stress-related process in relapse (Koob and Volkow, 2010, 2016). Finally, we found a decrease in the activity of the insula that reveals a dysregulation in the functioning of this region that has been shown to be critical for interoceptive processes and relapse (Goldstein et al, 2009).

\section{Methodological Considerations}

When interpreting the results of this study, several aspects should be considered. First, our measures reflect metabolic activity under resting states. Thus, it is possible that dysregulation in the ability of certain brain regions to respond to external or internal stimuli may have passed unnoticed in the present study. This is particularly important because most of the recent studies in humans have used fMRI approaches to highlight the changes in reactivity of the brain of individuals with addiction to several manipulations such as exposure to drug cues (Goldstein and Volkow, 2002; Volkow and Baler, 2014; Wilson et al, 2004). In addition, PET imaging allows a resolution of one millimeter (Caprioli et $a l, 2013$ ). For this reason, some regions that were too small to be identified individually, such as the SN/VTA or different nuclei of the Amyg, had to be pooled. Thus, it is possible that certain changes attributed to one region may be actually due to changes in one of their sub-regions. Conversely, it is possible that lack of effects in some regions may be in part due to differences in the activity of the sub-regions. Whereas in this study we focused our attention to the abstinence phase of addiction, brain-imaging studies could be potentially used longitudinally to compare brain functioning in the same individual before, during, and after exposure to cocaine. Unfortunately, because the behavioral equipment and the PET scan were not in the same location, we were not able to perform such studies. Future studies will be needed to allow this additional important comparison. Another important consideration is that given that imaging procedure was conducted twice in each rat, the effects of repeated manipulations such as fasting, habituation, and general anesthesia may have at least in part contributed to the effects measured in the second scan. However, analysis of brain activity in control rats at different time points did not show significant effect of retesting (data not shown). A final aspect to consider is that in our study, control naive animals did not undergo surgery and were not exposed to operant cages. While we cannot rule out the possibility that these manipulations may have contributed to differences in metabolic activity measured in ShA and LgA rats $v s$ controls, the contribution of these experimental differences is likely limited compared to exposure to cocaine. Furthermore, it should be considered that from a translational point of view, 
cocaine naive individuals do not share with individuals with cocaine addiction a wide variety of drug-related experiences and may indeed resemble in many ways to our naive control groups. Finally, whereas the comparison to naive control is an important aspect of this work, our main focus concerns the differences between ShA and LgA rats that had very similar experimental histories.

\section{CONCLUSIONS}

We show that extended access intake of cocaine in the escalation model (Ahmed and Koob, 1998) produce a complex regional and temporal pattern of changes in basal brain metabolic activity that are strikingly similar to those reported in humans and framed into the I-RISA model of addiction (Goldstein and Volkow, 2002). These functional dysregulations would lead to increased sensitivity to drugs and drug-related cues, difficulties in regulating emotional responses and deficits in cognitive functions that render individuals with addiction persistently vulnerable to relapse. The combination of animal models of addiction and brain imaging approaches represents a unique tool to investigate the brain mechanisms underlying the effects of novel therapeutic interventions.

\section{FUNDING AND DISCLOSURE}

This study was funded by the INSERM, the University of Poitiers, the University of Tours and the Fondation pour la Recherche Medicale (FRM, DPA20140629806 grant to MS). C. Nicolas was a recipient of a PhD fellowship by the PoitouCharentes region. The authors declare no conflict of interest.

\section{ACKNOWLEDGMENTS}

We thank Yavin Shaham and Mike Michaelides for helpful suggestions on a previous version of the manuscript. We would like to thank Cyclopharma Laboratories for providing ${ }^{18}$ FDG.

\section{REFERENCES}

Ahmed SH, Koob GF (1998). Transition from moderate to excessive drug intake: change in hedonic set point. Science 282: 298-300.

Arroyo M, Baker WA, Everitt BJ (2000). Cocaine selfadministration in rats differentially alters mRNA levels of the monoamine transporters and striatal neuropeptides. Brain Res Mol Brain Res 83: 107-120.

Calipari ES, Beveridge TJ, Jones SR, Porrino LJ (2013). Withdrawal from extended-access cocaine self-administration results in dysregulated functional activity and altered locomotor activity in rats. Eur J Neurosci 38: 3749-3757.

Caprioli D, Fryer TD, Sawiak SJ, Aigbirhio FI, Dalley JW (2013). Translating positron emission tomography studies in animals to stimulant addiction: promises and pitfalls. Curr Opin Neurobiol 23: 597-606.

Chen BT, Bowers MS, Martin M, Hopf FW, Guillory AM, Carelli RM et al (2008). Cocaine but not natural reward selfadministration nor passive cocaine infusion produces persistent LTP in the VTA. Neuron 59: 288-297.

Cosme CV, Gutman AL, LaLumiere RT (2015). The dorsal agranular insular cortex regulates the cued reinstatement of cocaine-seeking, but not food-seeking, behavior in rats. Neuropsychopharmacology 40: 2425-2433.

Fuchs RA, Evans KA, Ledford CC, Parker MP, Case JM, Mehta RH et al (2005). The role of the dorsomedial prefrontal cortex, basolateral amygdala, and dorsal hippocampus in contextual reinstatement of cocaine seeking in rats. Neuropsychopharmacology 30: 296-309.

Fuchs RA, Feltenstein MW, See RE (2006). The role of the basolateral amygdala in stimulus-reward memory and extinction memory consolidation and in subsequent conditioned cued reinstatement of cocaine seeking. Eur J Neurosci 23: 2809-2813.

Garcia-Fuster MJ, Flagel SB, Mahmood ST, Watson SJ, Akil H (2012). Cocaine withdrawal causes delayed dysregulation of stress genes in the hippocampus. PLoS ONE 7: e42092.

Goldstein RZ, Craig AD, Bechara A, Garavan H, Childress AR, Paulus MP et al (2009). The neurocircuitry of impaired insight in drug addiction. Trends Cogn Sci 13: 372-380.

Goldstein RZ, Volkow ND (2002). Drug addiction and its underlying neurobiological basis: neuroimaging evidence for the involvement of the frontal cortex. Am J Psychiatry 159: $1642-1652$.

Gould RW, Duke AN, Nader MA (2014). PET studies in nonhuman primate models of cocaine abuse: translational research related to vulnerability and neuroadaptations. Neuropharmacology 84: 138-151.

Gozzi A, Tessari M, Dacome L, Agosta F, Lepore S, Lanzoni A et al (2011). Neuroimaging evidence of altered fronto-cortical and striatal function after prolonged cocaine self-administration in the rat. Neuropsychopharmacology 36: 2431-2440.

Grimm JW, Hope BT, Wise RA, Shaham Y (2001). Neuroadaptation. Incubation of cocaine craving after withdrawal. Nature 412: 141-142.

Grimm JW, Lu L, Hayashi T, Hope BT, Su TP, Shaham Y (2003). Time-dependent increases in brain-derived neurotrophic factor protein levels within the mesolimbic dopamine system after withdrawal from cocaine: implications for incubation of cocaine craving. J Neurosci 23: 742-747.

Grimm JW, See RE (2000). Dissociation of primary and secondary reward-relevant limbic nuclei in an animal model of relapse. Neuropsychopharmacology 22: 473-479.

Hammer RP Jr., Pires WS, Markou A, Koob GF (1993). Withdrawal following cocaine self-administration decreases regional cerebral metabolic rate in critical brain reward regions. Synapse 14: 73-80.

Hanlon CA, Beveridge TJ, Porrino LJ (2013). Recovering from cocaine: insights from clinical and preclinical investigations. Neurosci Biobehav Rev 37(9 Pt A): 2037-2046.

Hearing MC, See RE, McGinty JF (2008). Relapse to cocaine-seeking increases activity-regulated gene expression differentially in the striatum and cerebral cortex of rats following short or long periods of abstinence. Brain Struct Funct 213: 215-227.

Howell LL (2008). Nonhuman primate neuroimaging and cocaine medication development. Exp Clin Psychopharmacol 16: 446-457.

Koob GF, Volkow ND (2010). Neurocircuitry of addiction. Neuropsychopharmacology 35: 217-238.

Koob GF, Volkow ND (2016). Neurobiology of addiction: a neurocircuitry analysis. Lancet Psychiatry 3: 760-773.

Lee BR, Ma YY, Huang YH, Wang X, Otaka M, Ishikawa M et al (2013). Maturation of silent synapses in amygdala-accumbens projection contributes to incubation of cocaine craving. Nat Neurosci 16: 1644-1651.

Lu L, Dempsey J, Shaham Y, Hope BT (2005a). Differential longterm neuroadaptations of glutamate receptors in the basolateral and central amygdala after withdrawal from cocaine selfadministration in rats. J Neurochem 94: 161-168.

Lu L, Grimm JW, Shaham Y, Hope BT (2003). Molecular neuroadaptations in the accumbens and ventral tegmental area during the first 90 days of forced abstinence from cocaine self-administration in rats. J Neurochem 85: 1604-1613. 
Lu L, Hope BT, Dempsey J, Liu SY, Bossert JM, Shaham Y (2005b). Central amygdala ERK signaling pathway is critical to incubation of cocaine craving. Nat Neurosci 8: 212-219.

Macey DJ, Rice WN, Freedland CS, Whitlow CT, Porrino LJ (2004). Patterns of functional activity associated with cocaine selfadministration in the rat change over time. Psychopharmacology (Berl) 172: 384-392.

McFarland K, Kalivas PW (2001). The circuitry mediating cocaineinduced reinstatement of drug-seeking behavior. J Neurosci 21: 8655-8663.

Noonan MA, Choi KH, Self DW, Eisch AJ (2008). Withdrawal from cocaine self-administration normalizes deficits in proliferation and enhances maturity of adult-generated hippocampal neurons. J Neurosci 28: 2516-2526.

Parvaz MA, Alia-Klein N, Woicik PA, Volkow ND, Goldstein RZ (2011). Neuroimaging for drug addiction and related behaviors. Rev Neurosci 22: 609-624.

Phelps ME, Huang SC, Hoffman EJ, Selin C, Sokoloff L, Kuhl DE (1979). Tomographic measurement of local cerebral glucose metabolic rate in humans with (F-18)2-fluoro-2-deoxy-D-glucose: validation of method. Ann Neurol 6: 371-388.

Pickens CL, Airavaara M, Theberge F, Fanous S, Hope BT, Shaham Y (2011). Neurobiology of the incubation of drug craving. Trends Neurosci 34: 411-420.

Pomierny-Chamiolo L, Miszkiel J, Frankowska M, Pomierny B, Niedzielska E, Smaga I et al (2015). Withdrawal from cocaine self-administration and yoked cocaine delivery dysregulates glutamatergic mGlu5 and NMDA receptors in the rat brain. Neurotox Res 27: 246-258.

Schiffer WK, Mirrione MM, Biegon A, Alexoff DL, Patel V, Dewey SL (2006). Serial microPET measures of the metabolic reaction to a microdialysis probe implant. J Neurosci Methods 155: $272-284$.

Sokoloff L (1977). Relation between physiological function and energy metabolism in the central nervous system. $J$ Neurochem 29: $13-26$.

Thompson AM, Swant J, Gosnell BA, Wagner JJ (2004). Modulation of long-term potentiation in the rat hippocampus following cocaine self-administration. Neuroscience 127: 177-185.

Torregrossa MM, Gordon J, Taylor JR (2013). Double dissociation between the anterior cingulate cortex and nucleus accumbens core in encoding the context versus the content of pavlovian cocaine cue extinction. J Neurosci 33: 8370-8377.

Volkow ND, Baler RD (2014). Addiction science: uncovering neurobiological complexity. Neuropharmacology 76 Pt B: 235-249.

Volkow ND, Fowler JS, Wang GJ (2003). The addicted human brain: insights from imaging studies. J Clin Invest 111: 1444-1451.

Volkow ND, Fowler JS, Wang GJ, Hitzemann R, Logan J, Schlyer DJ et al (1993). Decreased dopamine D2 receptor availability is associated with reduced frontal metabolism in cocaine abusers. Synapse 14: 169-177.

Volkow ND, Fowler JS, Wolf AP, Hitzemann R, Dewey S, Bendriem B et al (1991). Changes in brain glucose metabolism in cocaine dependence and withdrawal. Am J Psychiatry 148: 621-626.

Volkow ND, Fowler JS, Wolf AP, Schlyer D, Shiue CY, Alpert R et al (1990). Effects of chronic cocaine abuse on postsynaptic dopamine receptors. Am J Psychiatry 147: 719-724.

Volkow ND, Hitzemann R, Wang GJ, Fowler JS, Wolf AP, Dewey SL et al (1992). Long-term frontal brain metabolic changes in cocaine abusers. Synapse 11: 184-190.

Volkow ND, Wang GJ, Fischman MW, Foltin RW, Fowler JS, Abumrad NN et al (1997a). Relationship between subjective effects of cocaine and dopamine transporter occupancy. Nature 386: $827-830$.

Volkow ND, Wang GJ, Fowler JS (1997b). Imaging studies of cocaine in the human brain and studies of the cocaine addict. Ann N Y Acad Sci 820: 41-54, discussion 54-45.

Volkow ND, Wang GJ, Fowler JS, Logan J, Gatley SJ, Hitzemann R et al (1997c). Decreased striatal dopaminergic responsiveness in detoxified cocaine-dependent subjects. Nature 386: 830-833.

Wilson SJ, Sayette MA, Fiez JA (2004). Prefrontal responses to drug cues: a neurocognitive analysis. Nat Neurosci 7: 211-214.

Zavala AR, Biswas S, Harlan RE, Neisewander JL (2007). Fos and glutamate AMPA receptor subunit coexpression associated with cue-elicited cocaine-seeking behavior in abstinent rats. Neuroscience 145: 438-452.

Zorrilla EP, Valdez GR, Weiss F (2001). Changes in levels of regional CRF-like-immunoreactivity and plasma corticosterone during protracted drug withdrawal in dependent rats. Psychopharmacology (Berl) 158: 374-381.

Supplementary Information accompanies the paper on the Neuropsychopharmacology website (http://www.nature.com/npp). 